\title{
Granulomatosis with polyangiitis: rapidly progressive necrotizing glomerulonephritis in a pediatric patient
}

This article was published in the following Dove Press journal: International Journal of Nephrology and Renovascular Disease 23 April 2014

Number of times this article has been viewed

\author{
Mariana Luna' \\ Victoria Bocanegra ${ }^{3}$ \\ Patricia G Vallés ${ }^{1,2}$ \\ 'Nephrology Division, Pediatric \\ Department, Dr Humberto Notti \\ Pediatric Hospital, Mendoza, \\ Argentina; ${ }^{2}$ Pathophysiology Area, \\ Pathology Department, School of \\ Medicine, National Cuyo University, \\ Mendoza, Argentina; ${ }^{3}$ National Council \\ of Scientific and Technical Research of \\ Argentina (CONICET), Buenos Aires, \\ Argentina
}

Correspondence: Patricia G Vallés Pathiophysiology Area, Pathology Department, School of Medicine, National Cuyo University, Centro Universitario CP 5500, Mendoza, Argentina

Tel +5426I 4I3262 4

Fax +54 26I 4287370

Email pvalles@fcm.uncu.edu.ar

\begin{abstract}
Granulomatosis with polyangiitis (GPA) is associated with a broad range of clinical manifestations including renal disease. It is a systemic vasculitis that is rarely encountered in children. We present a 14-year-old girl who suffered from pharyngitis 1 week before admittance to hospital. She was admitted for macroscopic hematuria and oliguria, under the possibility of nephritic syndrome. Renal failure with rapidly progressive glomerulonephritis occurred within 24 hours. Immunologic tests showed the presence of type-C anti-neutrophil cytoplasmic antibodies (c-ANCA with antiproteinase 3 specificity) and renal biopsy revealed pauci-immune crescentic focal necrotizing glomerulonephritis. Treatment including methylprednisolone and cyclophosphamide intravenous pulses allowed renal recovery after 3 weeks. The clinical, hematological, and biochemical parameters improved substantially, achieving remission. Granulomatosis with polyangiitis, although rare in children, should be considered in the above clinical scenario. This case underlines that knowledge of renal histology diagnosis and early aggressive immunosuppressive therapy are essential for the management of these patients.
\end{abstract}

Keywords: acute renal failure, vasculitis, crescentic pauci-immune glomerulonephritis-Type-C, antineutrophil cytoplasmic antibodies (c-ANCA)-macroscopic hematuria

\section{Introduction}

Granulomatosis with polyangiitis (GPA), formerly called Wegener's granulomatosis, is a rare autoimmune disorder of unknown etiology that is characterized by granulomatous inflammation and antineutrophil cytoplasmatic antibodies (ANCA) associated with small vessel vasculitis. ANCA are mainly directed against proteinase 3 (PR3). GPA has a broad clinical spectrum that ranges from predominantly granulomatous manifestations restricted to the respiratory tract, to severe, life-threatening necrotizing vasculitis affecting many organs, with a predilection for lung and kidney involvement. The disease mostly affects adults aged $25-50$ years and is rare in children. ${ }^{1}$

The European classification criteria for granulomatosis with polyangiitis requires the diagnosis of three of the following six features: 1) abnormal urinalysis; 2) granulomatous inflammation on biopsy; 3) nasal sinus inflammation; 4) subglottic, tracheal, or endobronchial stenosis; 5) abnormal chest imaging; and 6) positive ANCA testing. ${ }^{2}$

\section{Case report}

The patient is a 14-year-old girl diagnosed with pharyngitis 1 week before admission. She was referred to the Dr Humberto Notti Pediatric Hospital for macroscopic hematuria accompanied by oliguria, with suspicion of nephritic syndrome. On examination, the patient appeared healthy with a body mass index of $22.5 \mathrm{~kg} / \mathrm{m}^{2}$, with 
no fever or edema. Her blood pressure was 110/90 mmHg (normal blood pressure levels for age, height and sex), with dispersed abdominal pain, aching shoulders, and myalgias. Family history was not significant and there was no history of illicit drugs.

Initial laboratory data were as follows: serum creatinine 3.10 $\mathrm{mg} / \mathrm{dL}$, urea $50 \mathrm{mg} / \mathrm{dL}$, hemoglobin concentration $10.3 \mathrm{~g} / \mathrm{dL}$, and urinalysis with more than 100 red blood cells, some of them dysmorphic without crystals or hyaline cylinders. Negative 24-hour proteinuria was reported. Laboratory data 12 hours after admission into hospital were as follows: serum creatinine $5.7 \mathrm{mg} / \mathrm{dL}$, urea $85 \mathrm{mg} / \mathrm{dL}$, leukocytes $12,500 / \mu \mathrm{L}$, platelet count $115 \times 10^{3} / \mu \mathrm{L}$, C-reactive protein (CRP) $2.27 \mathrm{mg} / \mathrm{dL}$ (227.3 mg/L), ferritin $313 \mathrm{ng} / \mathrm{mL}(6-70 \mathrm{ng} / \mathrm{mL})$, fibrinogen $476 \mathrm{mg} \%$ (200-400 mg\%), potassium $3.4 \mathrm{mEq} / \mathrm{L}$, sodium $133 \mathrm{mEq} / \mathrm{L}$, chloride $93 \mathrm{mEq} / \mathrm{L}$, calcium $8.59 \mathrm{mg} / \mathrm{dL}$, acid base balance $\mathrm{pH} 7.4 / \mathrm{PCO}_{2} 29.7 \mathrm{mmHg} / \mathrm{HCO}_{3} 20 \mathrm{mmol} / \mathrm{L}$, lactate dehydrogenase $800 \mathrm{U} / \mathrm{L}$, and decreased glomerular filtration rate $14.4 \mathrm{~mL} / \mathrm{m} / 1.73 \mathrm{~m}^{2}$ by Schwartz formula. Chest radiograph with diffuse interstitial infiltrate in both lungs was demonstrated. Renal ultrasound showed increased kidney size with increased renal parenchymal echogenicity consistent with parenchymal disease.

Twenty-four hours after admission, peritoneal dialysis was started. The next day, creatinine and uremia continued to increase. A percutaneous renal biopsy was performed and intravenous steroid pulses at $10 \mathrm{mg} / \mathrm{kg}$ /day were begun.

Serology tests demonstrated the presence of positive C-ANCA by indirect immunofluorescence with documented proteinase 3-specific ANCA (PR3-ANCA). P-ANCA and peroxidase-specific antineutrophil cytoplasmic autoantibodies (MPO-ANCA) were negative. Rheumatoid factor and C3, C4 complement levels were normal. Anti-DNA antibody titers measured by enzyme-linked immunosorbent assay (ELISA) and anti-nuclear antibody (ANA) by immunofluorescence were at normal levels. Viral serology (hepatitis B and C, HIV), and blood and throat cultures were negative. Anti-streptolysin $\mathrm{O}$ (ASO) titers were within the normal limits.

Diagnosis of renal involvement was confirmed through biopsy. Microscopic optic study in 20 glomeruli revealed renal parenchyma with less than $50 \%$ glomeruli presenting segmental capillary fibrinoid necrosis with neutrophil exudate surrounding glomeruli. Tubule interstitial infiltrate was also shown (Figure 1). Cellular crescent was seen in $20 \%$ of the glomeruli (Figure 2). Immunofluorescence with antibodies against IgG, IgA, IgM, and C3 was negative for immunoglobulins, and the intensity of immunofluorescence staining was very weak (trace or trace to 1+)

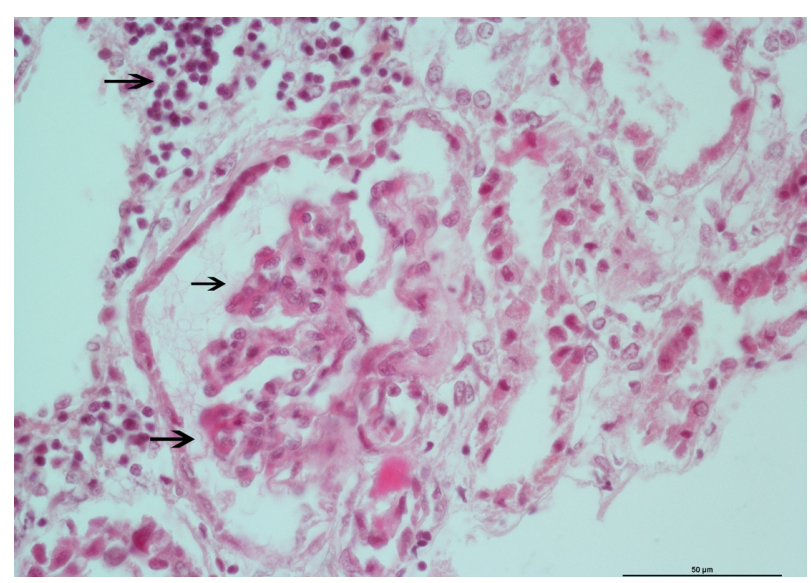

Figure I Renal biopsy specimen showing focal necrotizing glomerulonephritis in the patient.

Notes: Segmental capillary fibrinoid necrosis was present in glomeruli without crescents (middle and lower arrows) along with periglomerular and interstitial infiltration of mononuclear and neutrophil cells (upper arrow); hematoxylin and eosin stain. Original magnification $\times 400$.

for C3. The final diagnosis was focal segmental necrotizing glomerulonephritis.

To investigate other organs affected with small vessel vasculitis, nasal cavity and sinuses computer tomography (CT) showed rhinosinusitis signals and inflammatory tissue. Chest CT showed bilateral pleural effusion.

Immunosuppressive therapy was started with three methylprednisolone pulses, following by cyclophosphamide intravenous pulses (each with a dosage of $750 \mathrm{mg} / \mathrm{m}^{2}$ body surface) continued at monthly intervals for 12 months. After 23 days, the patient's renal function recovered. The early normalization of the glomerular filtration rate made plasma exchange in the protocol treatment unnecessary.

Further, the remission treatment consisted of a daily dose of mycophenolate mofetil $750 \mathrm{mg} / \mathrm{m}^{2}$ in two divided doses

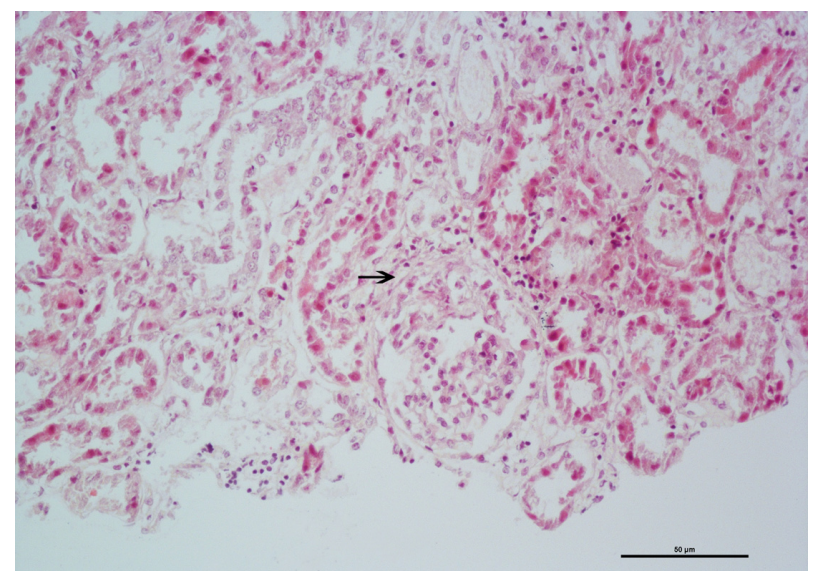

Figure 2 Renal biopsy specimen showing mild focal segmental extracapillary proliferation (arrow); hematoxylin and eosin stain.

Note: Original magnification $\times 200$. 
and oral prednisolone ( $1 \mathrm{mg} / \mathrm{kg}$ for 6 weeks) followed by slow tapering. She was discharged after 5 weeks. Neither symptoms nor signs of vasculitis were seen at the last clinical control. Six months after diagnosis, the patient was negative for PR3-ANCA.

\section{Discussion}

The present report describes the development of clinical rapidly progressive glomerulonephritis and histological crescentic necrotizing glomerulonephritis in a girl. Crescentic glomerulonephritis is classified into three main categories on the basis of direct immunofluorescence microscopy. Anti-glomerular basement membrane (GBM) crescentic glomerulonephritis is characterized by the presence of circulating antibodies to GBM and linear deposition of $\mathrm{IgG}$ along the GBM; immune complex-mediated crescentic glomerulonephritis is characterized by the deposition of immune complexes in the glomeruli; while pauci-immune crescentic glomerulonephritis presents as focal necrotizing glomerulonephritis with little or no glomerular staining for immunoglobulins. ${ }^{3}$

Rapid progressive renal failure was an initial clinical diagnosis in this patient who suffered from progressive renal impairment in a short period of time. Although immune complex-mediated crescentic glomerulonephritis is the most common cause of rapidly progressive glomerulonephritis in children, we unusually found renal histopathology of pauci-immune crescentic glomerulonephritis in this patient. These findings are also in contrast to previous case series in children showing that immune complex glomerulonephritis constitutes the majority of cases with crescentic glomerulonephritis. ${ }^{4}$

Importantly, renal biopsy with indirect immunofluorescent staining in the patient allowed the exclusion of antiGBM antibody disease in the setting of a pulmonary renal syndrome. Approximately three-fourths of patients with pauci-immune or ANCA-associated crescentic glomerulonephritis have systemic small-vessel vasculitis. Only ANCAs directed against PR3 or MPO have been associated with primary vasculitic syndromes. C-ANCA directed against PR3 is most specific for GPA as we have demonstrated in this patient.

However, pulmonary involvement occurred in half of the patients and subglottic stenosis is frequently present in pediatric patients at onset; these were not observed in our patient. Findings on chest radiography are abnormal in two-thirds of adults with GPA. Findings on thin-section sinus CT scans are abnormal in more than $90 \%$ of adults with GPA.
A similar number would be expected in the pediatric population, based on an $83 \%$ incidence of sinusitis. ${ }^{5}$

The clinical features identified in our patient as hematuria, slightly raised blood pressure, and the rapid loss of renal function suggest glomerulonephritis. In addition, with positive serum c-ANCA levels and kidney biopsy showing granulomatous inflammation, diagnosis of GPA can be confirmed.

In consideration of the pathogenesis, ANCA has been examined extensively ever since the soluble lysosomal enzymes myeloperoxidase and proteinase 3 were identified as targets. Antibodies to these enzymes activate primed neutrophils and cause neutrophil-dependent endothelial injury in vitro. However, the pathogenic potential of ANCA against proteinase 3, which is mainly found in patients with GPA remains unproven. Additionally, a subgroup of patients with pauci-immune crescentic glomerulonephritis persistently lack cANCA. ${ }^{6}$

Experimental studies have shown that proteinase-3directed autoimmunity involves the complementary peptide of proteinase 3, which is encoded by the antisense strand of the proteinase 3 gene. Exposure of the immune system to this peptide triggers the formation of antibodies that cross-react with proteinase 3 . DNA sequences complementary to the proteinase 3 gene have been identified in microorganisms, including Staphylococcus aureus, which supports the role of infectious agents as triggers of proteinase 3 autoimmunity via molecular mimicry. ${ }^{7,8}$ Recently, Kain et al reported that infection by fimbriated bacteria can trigger cross-reactive autoimmunity to a previously characterized ANCA antigen, lysosomal membrane protein 2 (LAMP-2), which results in the production of autoantibodies that activate neutrophils and damage human microvascular endothelium in vitro and in vivo, causing pauci-immune focal necrotizing glomerulonephritis, ${ }^{9}$ even though no infection was demonstrated by culture studies in our patient.

Our patient was treated with methylprednisolone and cyclophosphamide, which stabilized renal function after 3 weeks. Plasma exchange was not included in the protocol.

The optimal prescription of plasmapheresis for the treatment of GPA is uncertain. Clinical studies have focused on the use of plasma exchange to rescue organ function in rapidly progressive glomerulonephritis and lung hemorrhage, based on the rationale of removing circulating ANCAs. ${ }^{10}$ However, evidence derived from its use in children is scarce. ${ }^{11,12}$

Even in light of advances in the understanding of pathophysiology and serologic testing, renal biopsy remains the mainstay of the diagnosis of crescentic glomerulonephritis, 
and early definitive diagnosis of rapid progressive renal failure is essential to reverse the progression to end-stage kidney disease.

\section{Disclosure}

The authors report no conflicts of interest in this work.

\section{References}

1. Akikusa JD, Schneider R, Harvey EA, et al. Clinical features and outcome of pediatric Wegener's granulomatosis. Arthritis Rheum. 2007;57: 837-844.

2. Ozen S, Ruperto N, Dillon MJ, et al. EULAR/PReS endorsed consensus criteria for the classification of childhood vasculitides. Ann Rheum Dis. 2006;65(7):936-941.

3. Jennette J, Cohen J, Harringtom J, Madias N, Zusman C. Rapidly progressive crescentic glomerulonephritis. Kidney Int. 2003;63:1164-1177.

4. Dewan D, Gulati S, Sharma RK, et al. Clinical spectrum and outcome of crescentic glomerulonephritis in children in developing countries. Pediatr Nephrol. 2008;23(3):389-394.
5. Holle J, Laudien M, Gross W. Clinical manifestations and treatment of Wegener's Granulomatosis. Rheum Dis Clin North Am. 2010;36: $507-526$

6. Chen M, Kallenberg C, Zhao M. ANCA negative pauci-immune crescentic glomerulonephritis. Nat Rev Nephrol. 2009;5:313-318.

7. Bosch X, Mirapeix E. LAMP-2 illuminates pathogenesis of ANCA glomerulonephritis. Nat Rev Nephrol. 2009;5:247-249.

8. Pendergraft WF 3rd, Preston GA, Shah RR, et al. Autoimmunity is triggered by cPR-3105-3201, a protein complementary to human autoantigen proteinase-3. Nat Med. 2004;10:72-79.

9. Kain R, Exner M, Brandes R, et al. Molecular mimicry in pauciimmune focal necrotizing glomerulonephritis. Nat Med. 2008;14: 1088-1096.

10. Lapraik C, Watts R, Bacon P, et al. BSR and BHPR guidelines for the management of adults with ANCA associated vasculitis. Rheumatology (Oxford). 2007;46(10):1615-1616.

11. Wright E, Dillon MJ, Tullus K. Childhood vasculitis and plasma exchange. Eur J Pediatr. 2007;166(2):145-151.

12. Siomou E, Tramma D, Bowen C, Milford DV. ANCA-associated glomerulonephritis/systemic vasculitis in childhood: clinical featuresoutcome. Pediatr Nephrol. 2012;27(10):1911-2013.

\section{Publish your work in this journal}

The International Journal of Nephrology and Renovascular Disease is an international, peer-reviewed open-access journal focusing on the pathophysiology of the kidney and vascular supply. Epidemiology, screening, diagnosis, and treatment interventions are covered as well as basic science, biochemical and immunological studies. The journal welcomes original research, clinical studies, reviews \& evaluations, expert opinion and commentary, case reports and extended reports. The manuscript management system is completely online and includes a very quick and fair peerreview system, which is all easy to use. Visit http://www.dovepress.com/ testimonials.php to read real quotes from published authors. 